\title{
Inhibition of Retinal Pigment Epithelial Cell Senescence by Metformin: Implications for the Treatment of Macular Degeneration
}

\author{
Michael R. Kozlowski", Bryan Grossman, Alex Kneeland, Shelby Zemski, Abretia Crandell, Roni E. \\ Kozlowski \\ ${ }^{1}$ Arizona College of Optometry, Midwestern University, 19555 North 59th Avenue, Glendale, AZ 85308, USA
}

\begin{abstract}
A growing body of evidence suggests that the senescence of retinal pigment epithelial (RPE) cells contributes to the pathology of age-related macular degeneration (AMD). If confirmed, these data also suggest that agents that prevent or reduce cell senescence may be useful in the treatment of AMD. Metformin has been shown to inhibit the development of senescence in a number of cell types, although its effects of RPE cells have not yet been reported. A recent study showed that metformin also reduces the risk of AMD in patients taking this drug for the treatment of diabetes. In the present study we examine the effects of metformin on RPE cell senescence induced by oxidative stress. We report that metformin reduces two hallmarks of senescence in these cells: increased senescence-associated beta-galactosidase activity, and increased levels of $\mathrm{p} 21$. These results suggest that metformin may be useful in the treatment of AMD by inhibiting RPE cell senescence.
\end{abstract}

Keywords: Age-related macular degeneration, cell senescence, p21, retinal pigment epithelial cells, senescence-associated beta-galactosidase

\section{Introduction}

Cell senescence is a naturally occurring phenomenon characterized by a loss of mitotic potential and changes in cell structure and physiology that include altered cell morphology, increased activity of senescence-associated beta-galactosidase (SABG), altered patterns of gene expression and protein synthesis, and changes in the secretory phenotype [1-4]. Although cell senescence is thought to have important adaptive purposes, such as tumor suppression $[1,4,5]$, it can also disrupt the integrity and functioning of tissues containing senescent cells $[1,2,4,5]$.

One of the triggers for cell senescence is an excessive degree of telomere shortening $[1,4]$. This amount of shortening can be reached gradually through the small amount of DNA that is lost from the telomere with each round of cell division or rapidly under conditions such as oxidative stress in which the telomere may lose large amounts of DNA through DNA strand breaks $[1,4,6,7]$.
When telomeres become critically shortened, they are mistaken as damaged DNA by cell checkpoint mechanisms and a series of physiological events leading to senescence is initiated [1,4]. One critical step in the induction of cell senescence is an increase in levels of the cyclin-dependent kinase inhibitor, p21 [8,9]. Increased levels of p21 induce cell cycle arrest and cause changes in cell morphology and physiology that are characteristic of senescence including an increase in SABG activity $[10,11]$.

Corresponding Author: Michael R. Kozlowski, Arizona College of Optometry, Midwestern University, 19555 North 59th Avenue, Glendale, AZ 85308, USA Received date: November 09, 2020; Accepted date: November 24, 2020; Published date: November 25, 2020

DOI: https://doi.org/10.31546/2732-4516.1005 
Michael R. Kozlowski et al.

The disruption of tissue functioning caused by cell senescence has been proposed to contribute to several disorders associated with aging $[1,2,4,5]$. An increasing amount of evidence suggests that age-related macular degeneration (AMD) is one of these disorders [12,13]. Retinal pigment epithelium (RPE) cells undergo senescence both in culture and in situ, and the changes in cell physiology that accompany cell senescence are consistent with some of the pathologies seen in AMD [13-19]. For example, the disruption of lysosomal functioning indicated in senescent cells by increased levels of SABG activity may underly the development of macular drusen, a pathognomonic finding in AMD [20-22]. Thus, agents that inhibit RPE cell senescence may be useful in the prevention or treatment of AMD [13].

One of the agents that has been reported to have antisenescence effects is metformin [23-28]. Although this drug has been shown to inhibit senescence in a number of types of cells, RPE cells have not yet been examined. Recently, Chen et al. reported that metformin use in diabetics is associated with a lower risk of developing AMD [29]. The purpose of the present study is to determine whether metformin can also inhibit the development of senescence in RPE cells and may therefore, be useful in the prevention or treatment of AMD.

\section{Methods}

ARPE-19 cells were obtained from the American Type Culture Collection (ATCC; Manassas, VA). The cells were documented by ATCC as authentic ARPE-19 cells at passage 19. The cells were thawed and cultured according to the supplier's instructions. Briefly, the cells were rapidly thawed and then resuspended in DMEM:F12 medium (ATCC; Manassas, VA) supplemented with $10 \%$ fetal bovine serum (FBS; ATCC; Manassas, VA) in a tissue culture-treated T25 flask. They were grown and maintained in a $\mathrm{CO} 2$ incubator (Isotemp $^{\text {TM }}$, Fisher Scientific; Waltham, MA). We have previously found that ARPE-19 cells obtained at passage 19 begin to undergo senescence at between 40 and 58 populations doublings (approximately 12 to 20 passages) after having been received from the supplier [18]. In this study, therefore, cells were used within 7 passages after having been received to avoid replication-induced cell senescence.

Cell layers were dissociated with Trypsin-EDTA (MediaTech), counted using either a Countess ${ }^{\circledR}$ cell counter (Thermo Fisher;
Pittsburgh, PA) or a Moxi Z® cell counter (Orflo Technologies; Ketchum, ID), and checked for viability by trypan blue dye exclusion using the Countess ${ }^{\circledR}$ cell counter. The cells were then seeded in 6 well plates at a density of $2 \times 10^{4}$ cells per well.

Twenty-four hours after the cells had been seeded into the 6-well plates, metformin $(100 \mathrm{nM})$ or an equal amount of its vehicle (DMSO, 1:1000 dilution) was added to the culture medium. The cells were cultured for an additional 24 hours. The culture medium was then replaced by fresh medium without metformin or DMSO and t-butyl hydroperoxide (t-BHP; 50uM; Sigma Aldrich, Saint Louis, MO) or an equal volume of its vehicle $(10 \mu 1$ phosphate buffered saline) was added. After a period of one hour, the medium was again replaced with the medium containing either metformin or DMSO. The t-BHP exposure was repeated $24 \mathrm{~h}$ later. Twenty-four hours after the final $\mathrm{t}$ BHP treatment, levels of either p21 or SABG were measured.

Beta-galactosidase activity was measured according to the protocol of Dimri et al. [30]. The cells were washed in PBS, fixed for 3-5 minutes at room temperature in $2 \%$ formaldehyde $/ 0.2 \%$ glutaraldehyde, washed, and incubated at $37^{\circ} \mathrm{C}$ with fresh stain solution containing $1 \mathrm{mg}$ of 5-bromo-4-chloro-3indolyl P3-D-galactoside (X-Gal) per $\mathrm{ml}$ in $40 \mathrm{mM}$ citric acid/sodium phosphate, $\mathrm{pH} 6.0$, with $5 \mathrm{mM}$ potassium ferrocyanide, $5 \mathrm{mM}$ potassium ferricyanide, $150 \mathrm{mM} \mathrm{NaCl}$, and $2 \mathrm{mM} \mathrm{MgCl} 2$ (all chemical from Sigma Aldrich). After 24 hours of staining, the cells were washed with PBS and covered with glycerol as a preservative. The cells were photographed using a Moticam-5 digital camera attached to a Motic 160M inverted trinocular microscope (VWR, Radnor, PA). The same microscope and camera settings were used when photographic cells from each well in a given experiment.

The optical density of the staining of the 30 cells from the same area of each well was quantified using the GelAnalyzer ${ }^{\mathrm{TM}}$ software package. Cells whose staining was above the median level of the cells expo- 
-sed to t-BHP but not treated with metformin were considered "darkly staining".

Levels of p21 levels were measured using an Invitrogen ${ }^{\mathrm{TM}}$ p2 1 (Total) Human ELISA kit (Fisher Scientific, Pittsburgh, PA). The medium was removed from the well and the cells were rinsed twice with $200 \mu \mathrm{l}$ of $1 \mathrm{x}$ tris-buffered saline. The cells were then fixed in phosphate buffered saline containing $4 \%$ formaldehyde for 20 minutes at room temperature. They were then washed, quenched, and blocked using solutions provided with the kit. The cells were then incubated with an anti-p21 antibody for 16 hours at $4{ }^{\circ} \mathrm{C}$, washed, incubated with a HRPconjugated antibody recognizing the anti-p21 antibody for 1.5 hours, and washed again. Finally they were incubated with the kit's "Ready to Use" substrate for 30 minutes, the reaction stopped with a solution from also provided in the kit, and read at $450 \mathrm{~nm}$ using a plate reader (Synergy 2 ${ }^{\mathrm{TM}}$, Biotech, Winooski, VT). Protein levels were also measured on the same samples using Pierce ${ }^{\mathrm{TM}}$ BCA Protein Assay kit (Fisher Scientific), according to the provided protocol.

For statistical analyses, individual means were compared using t-tests. When multiple t-tests were done on the same data set, the probabilities were adjusted using the false discovery rate (FDR) method of Benjamini and Hochberg [31].

\section{Results}

Exposure of ARPE-19 cells to t-BHP for 1 hour on 2 successive days resulted in more than a doubling in the percentage of cells staining darkly for SABG (Figure 1). When the cells were treated with metformin $(100 \mathrm{nM})$ for the 24 hours periods before, between, and after the t-BHP exposures, but not during the exposures, the increase in darkly staining cells was reduced by approximately $70 \%$ (Figure 1). In the absence of t-BHP exposure, metformin produced no change in the percentage of cells darkly stained for SABG.

The same regimen of exposure of cells to t-BHP also produced increases in p21 levels of $141 \%$ and $211 \%$ in two separate experiments. Treatment of the cells with metformin, as above, reduced these increases by $10 \%$ and $14 \%$, respectively. In the absence of t-BHP exposure, metformin had a minimal effect on p21 levels, producing a $1 \%$ decrease, on average.

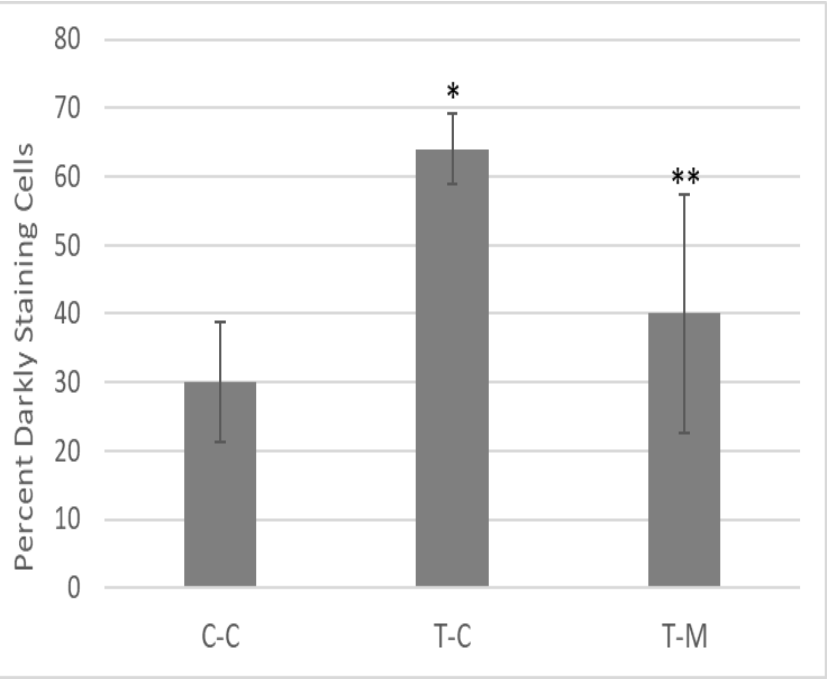

Figure 1: Effect of metformin treatment on the t-BHP-induced increase in SABG staining. The percentage of cells staining darkly for SABG (mean and standard errors) are shown under conditions of no t-BHP exposure or metformin treatment (C-C), exposure to tBHP but no metformin treatment (T-C), and both exposure to t$\mathrm{BHP}$ and treatment with metformin (T-M).

* Significantly different from C-C (t-test with FDR correction, $\mathrm{p}<$ $0.005, \mathrm{~N}=3$ )

** Significantly different from T-C (t-test with FDR correction, $\mathrm{p}$ $<0.05, \mathrm{~N}=3$ )

\section{Discussion}

The present study employed cells of the ARPE-19 human RPE cell line. There has been some controversy concerning whether this cell line truly represents natural RPE cells. Others and we have shown that the ARPE-19 cell line can be a reliable model of RPE cells if used under the proper culture conditions, and before the cells reach senescence, as was done in this study [18,32]. Senescence was induced in the ARPE-19 cells by exposure to the reactive oxygen species, t-BHP. Oxidative stress of cells produced by tBHP, as well as other means, has been shown to induce senescence in a number of types of cells, accompanied by increased SABG staining and elevated p21 levels [6,15,19,26,33-35].

Exposure of ARPE-19 cells to t-BHP for one hour on two successive days increased both the amount of SABG staining and the level of p21, consistent with the induction of senescence. These changes have also been found in RPE cells cultured directly from human 
retinas in response to oxidative stress produced by exposure to another reactive oxygen species, hydrogen peroxide [19]. When ARPE-19 cells were treated with metformin, the increases in both SABG staining and p21 levels were attenuated. The effectiveness of metformin in reducing these two key indicators of cell senescence agrees with previous results showing that metformin inhibits senescence in other types of cells, including senescence produced by reactive oxygen species [23-26].

The biochemical basis for the anti-senescence effects of metformin seen in this and other studies is not clear. Since metformin was not present during the periods that the cells were exposed to t-BHP in the present study it appears that metformin is not simply reducing the oxidative effectiveness of t-BHP itself. In addition, it has been shown that metformin does not reduce DNA damage in cells exposed to reactive oxidative species, thus it also may not be preventing telomere loss [36]. Several alternative mechanisms for the antisenescence effects of metformin have been proposed by others, and much additional work will be needed to determine which, or if any, of these are involved [23,24,26,36].

Even without completely understanding its mechanism of action, the ability of metformin to inhibit RPE cell senescence observed in the present study suggests that this drug should be studied further for its effectiveness in preventing or treating AMD.

Conflict of Interest: The authors declare no conflict of interest.

\section{References}

1. Rodier F, Campisi J. Four faces of cellular senescence. J Cell Biol. 2011;192:547-556.

2. Burton DG. Cellular senescence, ageing and disease. Age (Dordr). 2009;3:1-9.

3. Kuilman T, Chrysiis M, Wolter JM, Daniel SP. The essence of senescence. Genes Dev. 2010;24:2463-2479.

4. Munoz ED, Serrano M. Cellular senescence: from physiology to pathology. Nat Rev Mol Cell Biol. 2014;15:482-496.

5. Campisi J. Aging, cellular senescence, and cancer. Annu Rev Physiol. 2013;75:685-705.

6. von Zglinicki T, Saretzki G, Döcke W, Lotze C. Mild hyperoxia shortens telomeres and inhibits proliferation of - -fibroblasts: a model for senescence? Exp Cell Res. 1995;220:186-193.

7. von Zglinicki T. Oxidative stress shortens telomeres. Trends Biochem Sci. 2002;27:339-344.

8. Ben-Porath, I, Weinberg RA, The signals and pathways activating cellular senescence. Int $\mathbf{J}$ Biochem Cell Biol. 2005;37:961-976.

9. Herbig $U$, et al. Telomere shortening triggers senescence of human cells through a pathway involving ATM, p53, and p21(CIP1), but not p16(INK4a). Mol Cell. 2004;14:501-513.

10. Chen X, et al. Senescence-like changes induced by expression of p21(waf1/Cip1) in NIH3T3 cell line. Cell Res. 2002;12:229-233.

11. Shtutman M, et al. Cellular Model of p21-Induced Senescence. Methods Mol Biol. 2017;1534:31-39.

12. Blasiak $J$, et al. Cellular Senescence in AgeRelated Macular Degeneration: Can Autophagy and DNA Damage Response Play a Role? Oxid Med Cell Longev. 2017;2017:5293258.

13. Kozlowski, MR. RPE cell senescence: a key contributor to age-related macular degeneration. Med Hypotheses. 2012;78:505-510.

14. Shelton DN, et al. Microarray analysis of replicative senescence. Curr Biol. 1999;9:939-945.

15. Honda S, Hjelmeland LM, Handa JT. Senescence associated beta galactosidase activity in human retinal pigment epithelial cells exposed to mild hyperoxia in vitro. Br J Ophthalmol. 2002;86:159162.

16. Harman AM, et al. Development and aging of cell topography in the human retinal pigment epithelium. Invest Ophthalmol Vis Sci. 1997;38:2016-2026.

17. Burke JM, C Soref C. Topographical variation in growth in cultured bovine retinal pigment epithelium. Invest Ophthalmol Vis Sci. 1988;29:1784-1788.

18. Kozlowski MR, The ARPE-19 cell line: mortality status and utility in macular degeneration research. Curr Eye Res. 2015;40:501-509.

19. Yu AL, et al. Subtoxic oxidative stress induces senescence in retinal pigment epithelial cells via TGF-beta release. Invest Ophthalmol Vis Sci. 2009;50:926-935.

20. Lee BY, et al. Senescence-associated betagalactosidase is lysosomal beta-galactosidase. Aging Cell. 2006;5:187-195.

21. Kurz DJ, et al. Senescence-associated (beta)galactosidase reflects an increase in lysosomal mass during replicative ageing of human endothelial cells. J Cell Sci. 2000;113:3613-3622. 
Michael R. Kozlowski et al.

22. Kanski JJ. Age-related macular degeneration, in Clinical ophthalmology: A systematic approach. 2007, Elsevier: New York.

23. Smieszek A, et al. Antioxidant and Anti-Senescence Effect of Metformin on Mouse Olfactory Ensheathing Cells (mOECs) May Be Associated with Increased BrainDerived Neurotrophic Factor Levels-An Ex Vivo Study. Int J Mol Sci. 2017;18:872.

24. Moiseeva $\mathrm{O}$, et al. Metformin inhibits the senescenceassociated secretory phenotype by interfering with IKK/NF-kappaB activation. Aging Cell. 2013;12:489-498.

25. Chen $\mathrm{D}$, et al. Metformin protects against apoptosis and senescence in nucleus pulposus cells and ameliorates disc degeneration in vivo. Cell Death Dis. 2016;7:e2441.

26. Zhang C, et al. Metformin Prevents $\mathrm{H}(2) \mathrm{O}(2)$-Induced Senescence in Human Lens Epithelial B3 Cells. Med Sci Monit Basic Res. 2020; 26: e923391-1-e923391-9.

27. Molnar Z, et al. p21(WAF1/CIP1) Expression is Differentially Regulated by Metformin and Rapamycin. Int J Chronic Dis. 2014; 2014:327640.

28. Karnewar S, et al. Metformin regulates mitochondrial biogenesis and senescence through AMPK mediated H3K79 methylation: Relevance in age-associated vascular dysfunction. Biochim Biophys Acta Mol Basis Dis. 2018 Apr;1864(4 Pt A):1115-1128.

29. Chen YY, et al. Association between Metformin and a Lower Risk of Age-Related Macular Degeneration in Pat- -ients with Type 2 Diabetes. J Ophthalmol. 2019;2019:1649156.

30. Dimri GP, et al. A biomarker that identifies senescent human cells in culture and in aging skin in vivo. Proc Natl Acad Sci U S A. 1995;92:93639367.

31. Benjamini Y, Hochberg Y. Controlling the False Discovery Rate - a Practical and Powerful Approach to Multiple Testing. J R Stat Soc Series B Stat Methodol. 1995;57:289-300.

32. Dunn, KC, et al. ARPE-19, a human retinal pigment epithelial cell line with differentiated properties. Exp Eye Res. 1996;62:155-169.

33. Kaneto $\mathrm{H}$, et al. Oxidative stress induces p21 expression in pancreatic islet cells: possible implication in beta-cell dysfunction. Diabetologia. 1999;42:1093-1097.

34. Li YB, et al. Bisdemethoxycurcumin Increases Sirt1 to Antagonize t-BHP-Induced Premature Senescence in WI38 Fibroblast Cells. Evid Based Complement Alternat Med. 2013;2013:851714.

35. Wedel S, et al. tBHP treatment as a model for cellular senescence and pollution-induced skin aging. Mech Ageing Dev. 2020;190:111318.

36. Algire $\mathrm{C}$, et al. Metformin reduces endogenous reactive oxygen species and associated DNA damage. Cancer Prev Res (Phila). 2012;5:536-543. 